\title{
Population Pharmacokinetic-Pharmacodynamic Modeling of Inotersen, an Antisense Oligonucleotide for Treatment of Patients with Hereditary Transthyretin Amyloidosis
}

\author{
Rosie Z. Yu, Jon W. Collins, ${ }^{2}$ Shannon Hall, Elizabeth J. Ackermann, ${ }^{3}$ Richard S. Geary, \\ Brett P. Monia, ${ }^{1}$ Scott P. Henry, and Yanfeng Wang ${ }^{1}$
}

A population pharmacokinetic (PK) and pharmacodynamic (PD) model was developed for inotersen to evaluate exposure-response relationships and to optimize therapeutic dosing regimen in patients with hereditary transthyretin (TTR) amyloidosis polyneuropathy (hATTR-PN). Inotersen PK and TTR level (PD) data were composed of one Phase 1 study in healthy subjects, one Phase $2 / 3$ study in hATTR patients, and its one open-label extension study. Effects of intrinsic and extrinsic factors (covariates) on PK and PK/PD of inotersen were evaluated using a full model approach. Inotersen PK was characterized by a two-compartment model with elimination from the central compartment. The population PK analysis identified disease status and lean body mass (LBM) as significant covariates for inotersen PK. Nonetheless, the contribution of disease status and LBM on PK was small, as the difference in clearance $(\mathrm{CL} / F)$ was $11.1 \%$ between healthy subjects and patients with hATTR-PN and $38 \%$ between the lowest and highest LBM quartiles of the patient population. Age, race, sex, baseline renal function estimated glomerular filtration rate, and hepatic function markers (baseline albumin, bilirubin, and alanine aminotransferase values) were not statistically significant covariates affecting inotersen PK. An inhibitory effect indirect-response model (inhibition of TTR production) was used to describe the drug effect on TTR-time profiles, with baseline TTR included as a covariate. The overall population $I_{\max }$ and $\mathrm{IC}_{50}$, together with $95 \%$ confidence interval, was estimated to be $0.913(0.899-0.925)$ and $9.07(8.08-10.1) \mathrm{ng} / \mathrm{mL}$, respectively. V30M mutation showed no effect on the estimated $\mathrm{IC}_{50}$ value for hATTR patients. The final population PK and PK/PD model was used to simulate four different treatment regimens. The population PK/PD model developed well described the PK and PD of inotersen in patients with hATTR-PN and has been used for label recommendation and trial simulations.

Keywords: pharmacokinetics, pharmacodynamics, antisense, oligonucleotide, inotersen, hereditary TTR amyloidosis

\section{Introduction}

$\mathbf{H}$ EREDITARY TRANSTHYRETIN amyloidosis (hATTR) is a rare and fatal disease caused by mutations in the gene that codes for transthyretin (TTR) [1]. Single-point gene mutations destabilize the normal tetrameric structure of TTR protein causing its dissociation into free monomers and subsequent aggregation into insoluble extracellular deposits $[2,3]$. These deposits accumulate in multiple organs (including the peripheral nervous system, gastrointestinal tract, kidney, and heart) resulting in severe damage to cells, which causes the development of motor and sensory neuropathy, autonomic neuropathy, nephropathy, and cardiomyopathy [4]. On average, death occurs within $\sim 10$ years from symptom onset (but is shorter when significant cardiomyopathy is present) and is primarily due to malnutrition and cachexia, renal failure, and cardiac disease [4]. The only treatment option for hATTR was liver transplant, until the approval of two treatment options in 2018: patisiran (Onpattro ${ }^{\circledR}$; Alnylam Pharmaceuticals) and inotersen (Tegsedi ${ }^{\mathrm{TM}}$; Ionis Pharmaceuticals and Akcea Therapeutics), and both markedly changed the treatment landscape for this debilitating disease. Inotersen (also known as ISIS 420915) was approved by the European Commission, Canada, the U.S. Food and

\footnotetext{
${ }^{1}$ Ionis Pharmaceuticals, Inc., Carlsbad, California, USA.

${ }^{2}$ GlaxoSmithKline, Research Triangle Park, North Carolina, USA.

${ }^{3}$ Otonomy, Inc., San Diego, California, USA.
}

(c) Rosie Z. Yu et al. 2020; Published by Mary Ann Liebert, Inc. This Open Access article is distributed under the terms of the Creative Commons Attribution Noncommercial License (http://creativecommons.org/licenses/by-nc/4.0/) which permits any noncommercial use, distribution, and reproduction in any medium, provided the original author(s) and the source are cited. 
Drug Administration, and Brazil for the treatment of stage 1 or stage 2 polyneuropathy (PN) in adult patients with hATTR.

The strategy of treating hATTR patients with inotersen is to reduce the levels of mutated and wild-type TTR protein secreted by the liver, a primary organ for antisense oligonucleotide (ASO) distribution following systemic administrations. Inotersen is a $2^{\prime}-O$-(2-methoxyethyl) $\left(2^{\prime}\right.$-MOE) modified phosphorothioate oligonucleotide, which targets to the messenger RNA (mRNA) of human TTR via Watson-Crick base pairing and prevents expression of the encoded "disease-related" TTR protein. Inotersen demonstrated potent activity in vitro in reducing TTR mRNA levels in HepG2 cells and primary hepatocytes. Pharmacology studies conducted with inotersen in human TTR transgenic mice and cynomolgus monkeys demonstrated reproducible and robust reductions in liver TTR mRNA levels, which were accompanied by equally robust and significant reductions in plasma TTR protein levels.

The clinical experience included a Phase 1 double-blind, placebo-controlled, dose-escalation, first-in-human clinical study and a Phase $2 / 3$ pivotal study in hATTR patients with open-label extension (OLE) up to 5 years. In the Phase 1 study, dose- and exposure-dependent reductions in plasma TTR levels were observed with an acceptable safety and tolerability profile when administered subcutaneously (SC) to healthy subjects up to $400 \mathrm{mg}$ inotersen per week for 4 weeks. An exploratory pharmacokinetic (PK)/pharmacodynamic (PD) model was developed based on Phase 1 data [5] and was used to simulate TTR reduction at various dosing regimens. PK/PD simulations predicted that over $80 \%$ or $90 \%$ of patients would achieve TTR reduction of $>60 \%$ or $>50 \%$, respectively, following 3 months of $300 \mathrm{mg}$ inotersen once per week (QW). Therefore, the $300 \mathrm{mg}$ once weekly dosing regimen was selected for the pivotal Phase $2 / 3$ study. In the Phase 2/3 study in hATTR-PN patients, a $68 \%-74 \%$ mean (median: $75 \%-79 \%$ ) reduction in TTR levels was achieved from week 13 to 65 of treatment with $300 \mathrm{mg}$ inotersen QW and maintained throughout the 15-month treatment period in inotersentreated subjects, consistent with results obtained in the inotersen Phase 1 healthy volunteer study [6]. The robust and persistent TTR reductions in hATTR patients were accompanied by clinically and statistically significant benefit in favor of inotersen treatment, as demonstrated by both primary end points [modified Neuropathy Impairment Score (mNIS) +7 and Norfolk Quality of Life Diabetic Neuropathy (QoLDN)], as well as multiple prespecified sensitivity analyses, in multiple secondary, tertiary, and exploratory end points. Therefore, these results demonstrated that inotersen treatment provides clinical benefit to multiple aspects of the disease, including muscle weakness, large and small fiber sensation, and trends for benefit for autonomic symptoms. The overall safety profiles following chronic inotersen treatment were manageable. Taken together, these findings support the use of inotersen for the treatment of patients with hATTR.

The clinical PKs and PDs of inotersen have been evaluated in the first-in-human Phase 1 clinical study and the Phase 2/3 pivotal study with OLE by measuring plasma drug levels and serum TTR levels over time. The objective of this work was to develop a population PK and PK/PD model that describe the PKs and PDs of inotersen following SC administrations and evaluate the effect of intrinsic and extrinsic factors that potentially affect $\mathrm{PK}$ and PK/PD of inotersen and to perform alternative dose regimen simulations using the established PK/PD model.

\section{Materials and Methods}

\section{Test compounds}

Inotersen is a synthetic ASO of 20 nucleotides (ie, a 20mer) in length that are connected sequentially by phosphorothioate linkages, with a total of ten $2^{\prime}$-MOE modified ribofuranosyl nucleotides. The sequence of inotersen is: T ${ }^{\mathrm{M}}$ CTTG GTTA ${ }^{\mathrm{M}}$ CATGAA $\mathrm{AT}^{\mathrm{M}} \mathrm{C}^{\mathrm{M}} \mathrm{C}^{\mathrm{M}} \mathrm{C}$ with $2^{\prime}$-MOE modifications at positions 1-5 and 15-20 (underlined). In addition, all the cytosines of the compound were modified to contain a 5-methyl group (5-methyl cytosine, ${ }^{\mathrm{M}} \mathrm{C}$ ). The molecular formula of inotersen is $\mathrm{C}_{230} \mathrm{H}_{299} \mathrm{~N}_{69} \mathrm{O}_{121} \mathrm{P}_{19} \mathrm{~S}_{19} \mathrm{Na}_{19}$, and the molecular weight is $7600.8 \mathrm{amu}$. The molecular target of inotersen is within the $3^{\prime}$ untranslated region of the human TTR mRNA and binds to the mRNA using Watson and Crick base pairing.

Inotersen was formulated in water-for-injection $(200 \mathrm{mg} / \mathrm{mL})$ and supplied as a sterile solution in a 1 or $1.5 \mathrm{~mL}$ deliverable volume in a sealed glass vial or as a single-dose prefilled syringe in a $1.5 \mathrm{~mL}$ deliverable volume and contains no preservatives.

\section{Clinical studies}

All studies were performed according to the amended Declaration of Helsinki, and all patients provided written informed consent.

All three clinical studies conducted with inotersen by Ionis Pharmaceuticals were included in this population PK and PK/PD analysis.

The first study was a Phase 1 double-blind, placebocontrolled, dose-escalation study designed to assess the safety, tolerability, PKs, and PDs of single and multiple doses of inotersen administered SC to healthy subjects. Four singledose cohorts $(50,100,200$, and $400 \mathrm{mg}$ ) and five multipledose cohorts $(50,100,200,400$, and $300 \mathrm{mg}$ ) were evaluated sequentially with $3: 1$ or $8: 2$ randomization to inotersen or placebo, respectively. Subjects enrolled in the multiple-dose cohorts received a total of six doses of Study Drug (inotersen or placebo) administered by SC injection: three doses on alternate days during the first week (days 1, 3, and 5) and then once a week for the next 3 weeks (days 8, 15, and 22). All doses were administered as single SC injections with the exception of the $400 \mathrm{mg}$ doses, which were administered as two $200 \mathrm{mg}$ SC injections. The study enrolled 65 healthy volunteers (16 in the single-dose cohorts and 49 in the multiple-dose cohorts). Of the 65 subjects, 51 were administered inotersen and 14 were administered placebo.

Plasma samples for measurement of inotersen concentrations were collected at predose and 0.5, 1, 1.5, 2, 3, 4, 6, 8, 12, and $24 \mathrm{~h}$ following the SC injection on day 1 for the singledose cohorts and on days 1 and 22 for the multiple-dose cohorts. Plasma trough samples were collected before dose on days $3,5,8,15$, and 22 in the multiple-dose cohorts. In addition, post-treatment plasma samples for determination of elimination half-life were collected during the post-treatment follow-up period, on days 4 and 8 in the single-dose cohorts and on days 29, 36, 50, 64, 78, and 92 in the multiple-dose cohorts. Serum samples for TTR analysis (PD) was collected at screening, predose, $24 \mathrm{~h}$ postdose, and on days 4 and 8 in the single-dose cohorts and at predose and $24 \mathrm{~h}$ postdose on days $1,3,5,8,15$, and 22 and during post-treatment period on days 29, 36, 43, 50, 64, 78, and 92 in the multiple-dose cohorts. 
The second study was a pivotal Phase 2/3 randomized, double-blind placebo-controlled study to assess the efficacy and safety of inotersen in patients with hATTR. The primary objective was to evaluate the efficacy of inotersen compared to placebo, given for 65 weeks, as measured by the change from baseline in the mNIS +7 and in the Norfolk QoL-DN questionnaire total score, in patients with hATTR. Patients were randomized in a $2: 1$ ratio to receive $300 \mathrm{mg}$ inotersen or placebo. Study Drug (inotersen or placebo) was administered thrice on alternate days during week 1 (days 1,3 , and 5 ) and once weekly thereafter. A total of 172 subjects were dosed; 112 were administered inotersen and 60 received placebo. If required for safety or tolerability reasons, temporary dose reductions or interruptions were permitted. Plasma trough PK and PD samples were collected from all patients during the 65-week treatment phase and posttreatment follow-up period (if available). In addition, serial plasma samples were collected on days 1, 240, and 449 (weeks 1,35 , and 65, respectively) from a small number of subjects (a total of 10 subjects) at selected sites enrolled in the PK subgroup, for the assessment of inotersen PK in patients with hATTR.

The third study was an OLE study for eligible patients who had satisfactorily completed the pivotal Phase $2 / 3$ as described above, and patients received $300 \mathrm{mg}$ inotersen SC once weekly for up to 260 weeks (5 years) in the OLE study. Subjects who had a dose reduction or schedule change in the pivotal Phase $2 / 3$ study were permitted to continue with the adjusted dose level or schedule in the OLE study. A total of 108 subjects had at least one evaluable PK sample and were included in the analysis. Plasma trough PK and PD samples were collected periodically from patients in the OLE study.

There were total of 202 subjects in the study dataset, with $74.8 \%(N=151)$ of the analysis population being patients with hATTR and $25.2 \%(N=51)$ being healthy subjects (Table 1). Most individuals were Caucasian (87.6\%), with 7.43\% African Americans and 3.47\% Asians. There were more males $(69.8 \%)$ than females in the analysis population. There was no use of nonsteroidal anti-inflammatory drugs
(NSAID), antirheumatic products, or renin-angiotensin agent in the hATTR population; however, roughly a third of the hATTR population used diuretics $(30.5 \%)$ or antithrombotic agents (36.4\%), and $83.4 \%$ of the hATTR patients used nonNSAID analgesic agents. Healthy volunteers from the Phase 1 study were younger on average, had slightly higher baseline TTR levels, and had lower total bilirubin levels than hATTR patients in the pivotal Phase 2/3 study.

\section{Analytical methods}

Quantitation of inotersen concentrations in plasma. All plasma samples were analyzed using a quantitative, sensitive, hybridization-based enzyme-linked immunosorbent assay method, a variation on the method reported previously [7]. The assay was validated for precision, accuracy, selectivity, sensitivity, hook effect, metabolite cross-reactivity and interference, stability, and lack of antidrug antibody (ADA) interference in the quantitation of inotersen before analysis of the human plasma samples.

Plasma sample analyses were conducted at Covance Laboratories (West Trenton, NJ) and were performed based on the principles and requirements described in 21 CFR Part 58. The assay conducted with synthesized putative shortened oligonucleotide metabolite standards showed no measurable cross-reactivity, confirming the assay's specificity for inotersen. The quantitation range was $1.00-200 \mathrm{ng} / \mathrm{mL}$, with the lower and higher ends of the range defining the lower limit of quantitation (LLOQ) and upper limit of quantitation (ULOQ), respectively. There were no active metabolites for inotersen present in plasma.

Quantitation of TTR in serum. Serum samples for TTR (aka prealbumin) were analyzed by South Bend Medical Foundation (South Bend, IN) using a standardized immunoturbidimetric assay on Roche automated clinical chemistry analyzers. Anti-prealbumin antibodies reacted with the antigen in the sample to form antigen/antibody complexes, which were determined turbidimetrically after agglutination.

Table 1. Summary of Subject Demographics and Baseline Laboratory Values

\begin{tabular}{|c|c|c|c|c|c|c|}
\hline Parameter & Mean $(S D)$ & Minimum & Median & Maximum & Units & $N_{\text {Subjects }}$ \\
\hline Age & $55.4(13.8)$ & 25.0 & 57.0 & 81.0 & Years & 202 \\
\hline Height & $172(9.43)$ & 144 & 173 & 196 & $\mathrm{Cm}$ & 202 \\
\hline Ideal body weight & $66.2(10.0)$ & 38.1 & 68.3 & 89.2 & $\mathrm{Kg}$ & 202 \\
\hline Lean body mass & $51.7(8.44)$ & 31.3 & 51.6 & 80.3 & $\mathrm{Kg}$ & 202 \\
\hline Lean body weight & $54.8(10.0)$ & 31.4 & 54.9 & 82.9 & $\mathrm{Kg}$ & 202 \\
\hline Weight & $72.7(16.2)$ & 37.0 & 71.8 & 140 & $\mathrm{Kg}$ & 202 \\
\hline BMI & $24.6(4.45)$ & 13.3 & 24.5 & 40.2 & $\mathrm{~kg} / \mathrm{m}^{2}$ & 202 \\
\hline BSA & $1.85(0.223)$ & 1.30 & 1.83 & 2.61 & $\mathrm{~m}^{2}$ & 202 \\
\hline TTR & $22.0(5.95)$ & 3.80 & 22.1 & 39.7 & $\mathrm{mg} / \mathrm{dL}$ & 202 \\
\hline Albumin & 4,249 (314) & 3,167 & 4,233 & 5,000 & $\mathrm{mg} / \mathrm{dL}$ & 202 \\
\hline ALT & $23.1(10.8)$ & 6.00 & 20.7 & 83.2 & $\mathrm{U} / \mathrm{L}$ & 202 \\
\hline AST & $25.5(7.68)$ & 12.3 & 24.0 & 60.0 & $\mathrm{U} / \mathrm{L}$ & 202 \\
\hline Total bilirubin & $0.586(0.306)$ & 0.0800 & 0.517 & 2.18 & $\mathrm{mg} / \mathrm{dL}$ & 202 \\
\hline CrCL & $100(34.0)$ & 35.2 & 97.9 & 220 & $\mathrm{~mL} / \mathrm{min}$ & 202 \\
\hline eGFR & 93.2 (19.9) & 41.3 & 93.9 & 148 & $\mathrm{~mL} / \mathrm{min} / 1.73 \mathrm{~m}^{2}$ & 202 \\
\hline
\end{tabular}

$N_{\text {Subjects }}$ refers to the number of subjects over which the parameter statistics were computed and included five subjects who received treatment but had no PK observations.

PK, pharmacokinetic; BMI, body mass index; TTR, transthyretin; BSA, body surface area; ALT, alanine aminotransferase; AST, aspartate aminotransferase; CrCL, creatinine clearance; eGFR, estimated glomerular filtration rate. 
The quantitation range of the assay was from 3 to $80 \mathrm{mg} / \mathrm{dL}$ (ie, $30-800 \mathrm{ng} / \mathrm{mL}$ ), with the lower and higher ends of the range defining the LLOQ and ULOQ, respectively.

\section{Data analyses}

A population model using nonlinear mixed-effects modeling software, NONMEM ${ }^{\circledR}$ (version 7.2; ICON Plc, Hanover, MD), was developed to quantitatively characterize the PKs and PDs of inotersen to evaluate effects of covariates on inotersen PK/PD and to determine the inter- and intrasubject variability. A sequential modeling approach was used where a population-based PK model was established first to describe the kinetics of inotersen and, subsequently, using the post hoc PK as input to develop the population-based PD model. The first order conditional estimation method with interaction was used throughout model development.

Model features (eg, lag times, compartments, covariances, and so on) were added or removed based on evaluation of diagnostic plots, consideration of biological plausibility, and statistical tests. For hierarchical (or nested) models, the significance of adding or removing a parameter was assessed using the likelihood ratio test. For these models, the difference in the objective function value $(\triangle \mathrm{OFV})$ is $\sim \chi^{2}$ distributed with $\mathrm{n}$ degrees of freedom. As such, considering a significance level $(\alpha)$ of 0.05 , the proposed change to the model was considered to improve the model fit only if $\Delta \mathrm{OFV}$ is at least 3.84 when $n=1$ and 5.99 for $n=2$. In contrast, for non-nested models, the Akaike Information Criterion (AIC) and/or the Bayesian Information Criterion (BIC) were used to compare models following the principle that the lower the $\mathrm{AIC}$ and/or BIC, the better the model.

\section{Population PK modeling}

The population PK analysis included pooled data from three clinical studies in healthy volunteers and patients with hATTR-PN, as described above. The analysis dataset consisted of 202 subjects ( 51 healthy volunteers and 151 patients with hATTR-PN), among which 5 patients with hATTR-PN did not have PK data yet at the time of analysis. Therefore, available PK data from 197 subjects (51 healthy volunteers and 146 patients with hATTR-PN) with a total of 3,602 observations were used for the population PK analysis, to estimate the population PK parameters and the associated intersubject variability and residual error, to identify significant covariates, and to estimate their impact on exposure.

After visual inspection of the PK data following SC administration, plasma inotersen concentrations declined in a biphasic manner with time (Supplementary Fig. S1). In addition, one, two, and three-compartment models were fit to the naive pooling of all available data, and the twocompartment model was confirmed as the most appropriate base model structure for inotersen population PK analysis. The model was parameterized for absorption rate constant $\left(K_{\mathrm{a}}\right)$, clearance $(\mathrm{CL})$, central volume of distribution $\left(V_{\mathrm{c}}\right)$, intercompartmental clearance $(Q)$, and peripheral volume of distribution $\left(\mathrm{V}_{\mathrm{p}}\right)$ (Fig. 1) and included interindividual variability (IIV) on CL, $V_{\mathrm{c}}$, and $V_{\mathrm{p}}$, with additive residual error model on log transformed data.

The dose nonlinearity on clearance was observed in the Phase 1 data [5]. Thus, the dose nonlinearity was modeled by increasing the central clearance as the dose decreased using a

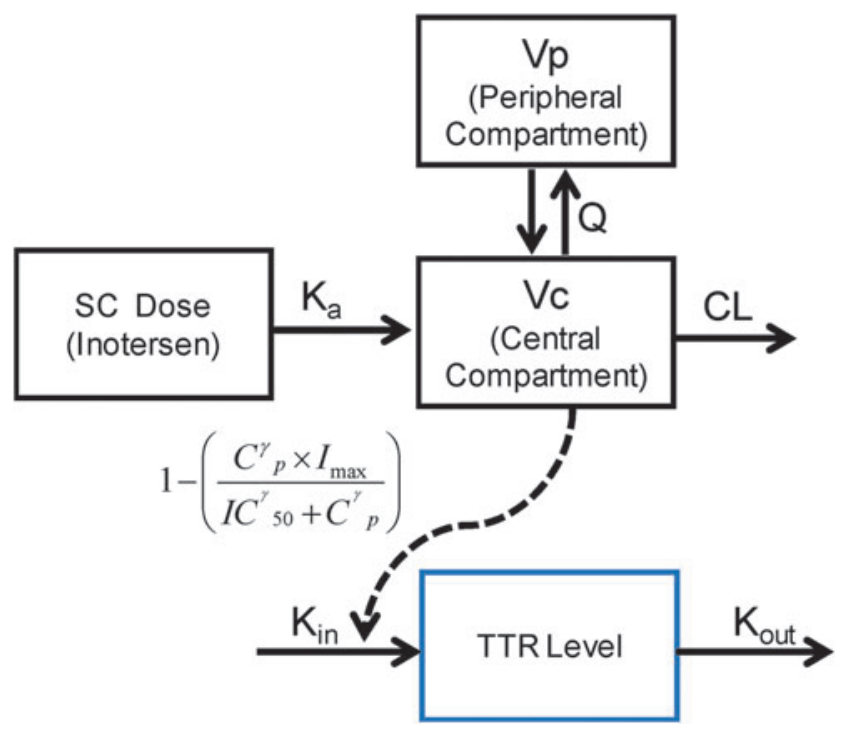

FIG. 1. Base PK and PK-PD model schematic. PK, pharmacokinetic; PD, pharmacodynamic.

simplified exponential model with dose administered being normalized to $300 \mathrm{mg}$ dose.

Following the identification of the base model, population PK covariate relationships were systematically examined using the Gastonguay full model approach [8], with backward elimination of candidate covariates. The covariates assessed were body size [body weight, lean body mass (LBM), body surface area, and body mass index], age effects ( $>65$ years old), sex, race, baseline estimated glomerular filtration rate (eGFR), baseline albumin, baseline bilirubin normalized by upper limit of normal (ULN), baseline alanine aminotransferase (ALT), baseline TTR, TTR V30M mutation, disease state, frequently used concomitant medications ( $\geq 20 \%$ of population), including diuretics, antithrombotic, and non-NSAID analgesics, and region of the world from which the participants originated from.

ADAs were detected following chronic treatment with inotersen and were characterized by a late onset (median onset $>6$ months) and low antibody titers (median peak titer $\leq 800$ ) in the pivotal Phase $2 / 3$ and OLE studies (internal data). The presence of ADA affected plasma trough concentrations of inotersen ( $\left.C_{\text {trough }}\right)$ to various degrees; however, the presence of ADA did not change other PK parameters (including $C_{\mathrm{max}}$, area under the curve [AUC], $t_{1 / 2 \lambda_{\mathrm{z}}}$, and $\mathrm{CL}_{\mathrm{ss}} /$ $F)$. In addition, the presence of ADA did not have any effect on PD (serum TTR levels), clinical primary efficacy end points (mNIS +7 and Norfolk QoL-DN Score), nor incidence or seriousness of all safety measures evaluated. These findings suggest that anti-inotersen antibodies are binding antibodies but not neutralizing antibodies and did not affect pharmacological response, clinical benefit, or safety of inotersen. Because of these reasons, the PK data collected post the onset of ADA were excluded from the population PK model, in an effort to develop a population PK model and characterize potential effects of various covariates without confounding effects from ADA.

Candidate models were compared with state-of-the-art diagnostics, the selected final PK model was qualified with visual predictive checks, and the precision of the final model 
parameters was estimated using covariance and nonparametric bootstrap analyses.

\section{Population PD modeling}

The population PD model of inotersen was developed with the aim to understand the changes in TTR response following the administration of inotersen in patients with hATTR. Based on the mechanism of action of inotersen as described previously, the counter-clockwise hysteresis loop of the timeordered TTR level versus inotersen plasma trough concentrations and visual inspection of the TTR over time data following SC administration (Supplementary Fig. S2), an indirect response model with an inhibition function on the input rate was used as the base PD model to describe the effect of inotersen treatment on serum TTR levels:

$$
\frac{d T T R}{d t}=k_{\text {in }}\left(1-\frac{I_{\max } C_{\mathrm{p}}}{I C_{50}+C_{\mathrm{p}}}\right)-k_{\mathrm{out}} T T R
$$

Where $k_{\text {in }}$ and $k_{\text {out }}$ represent the zero-order synthesis rate and first order turnover of TTR, respectively. $I_{\max }$ and $\mathrm{IC}_{50}$ describe the maximum inhibition of TTR and plasma concentration of inotersen corresponding to $50 \%$ of maximum drug effect, respectively.

The population PD model was developed following an iterative model building process using a nonlinear mixed effects approach. Modeling was performed on log-transformed TTR data, which were proved to be more stable and led to more consistent model convergence. Individual PK parameters obtained from the final population PK model were used to generate individual drug input for the PK/PD model. Covariates evaluated for the PD model included disease status (healthy volunteers vs. hATTR patients), baseline TTR levels, and TTR mutation (V30M and non-V30M). Final PD model was qualified with visual predictive checks, and the precision of the final model parameters was estimated using covariance and nonparametric bootstrap analyses.

\section{Simulations}

Simulations were conducted based on the final population PK and PD model to simulate concentration-time profiles of inotersen and TTR, as well as steady-state exposure PK parameters in patients with hATTR for the following dosing regimens:

- $300 \mathrm{mg}$ QW for 65 weeks

- $300 \mathrm{mg}$ once every other week (QOW) for 65 weeks

- $150 \mathrm{mg}$ QW for 65 weeks

- $300 \mathrm{mg}$ with loading dose on days 1,3 , and 5 during the first week, followed by QW for an additional 64 weeks

A total of 500 replicated datasets were produced for each simulation, with parameter uncertainty included using the variance-covariance matrix from the final model as the prior distribution. In each replicated dataset, the 151 patients from the original dataset were used along with their demographic variables, thus total of 75,500 patients were simulated. Steadystate exposure measures $\left(\mathrm{AUC}_{0-\tau}, C_{\max }, C_{\text {trough }}\right)$ from these simulations were summarized by geometric mean $[90 \%$ confidence interval (CI)] following the first dose on day 1 and the last dose after 65 weeks of dosing. The predicted TTR levels from these simulations were plotted as median (90\% prediction interval $[\mathrm{PI}])$.

\section{Results}

\section{Population PK model}

Following the identification of the base model as a twocompartment linear model with first order absorption following SC administration and first order elimination from the central compartment, dose nonlinearity term was added, which resulted in a decrease in the objective function of 57.12 points, as well as a reduction of IIV in CL/F, $V_{\mathrm{c}} / F$, and $V_{\mathrm{p}} / F$. The final population PK model suggests that the apparent dose nonlinearity is driven by a higher plasma clearance (primarily distributional clearance) at the lowest dose level of $50 \mathrm{mg}$. This apparent nonlinearity, however, will not be clinically relevant because the projected clinical dose levels are in the range of $150-300 \mathrm{mg}$.

Then, a full covariate approach followed by stepwise backward elimination was undertaken, incorporating remaining predetermined covariates, and the full covariate model was then subject to backward elimination process to exclude statistically and clinically insignificant covariates. The effect of four different measures of body size (body weight, LBM, body surface area, and body mass index) on the clearance and central volume of distribution was evaluated individually. LBM was chosen as the measure of body size based on the largest drop in the OFV. The model included interindividual variability on clearance, central volume, and peripheral volume and with additive residual error model on log transformed data. As shown in Figs. 2 and 3, only LBM and disease state showed clinical significance and remained in the model, where LBM remained as covariate on the central and intercompartmental clearances and the central and peripheral volumes and disease status on the central compartment clearance and central volume.

Bayesian post hoc estimates of exposures showed that the difference in the steady-state $\mathrm{AUC}_{\tau}$ or $C_{\max }$ was small between LBM quartile categories. For example, the geometric mean ratio for steady-state $\mathrm{AUC}_{\tau}$ and $C_{\max }$ was 1.38 and 1.34, respectively, between the first quartile (Q1) of LBM (LBM in the range of $31.1-45.7 \mathrm{~kg}$ ) and the fourth quartile (Q4) (LBM in the range of $56.0-80.3 \mathrm{~kg}$ ). The difference in geometric mean of $C_{\text {trough }}$ level between Q1 and Q4 was even less, $\sim 10 \%$. Similarly, small differences $(<30 \%)$ in the exposure are observed based on body weight quartile categories (Q1 in the range of $37.0-58.2 \mathrm{~kg}$ vs. Q4 in the range of 80.8$140 \mathrm{~kg}$ ). Thus, overall the effect of body weight or LBM on inotersen exposure was considered to be small and may not be clinically relevant.

There were differences in some of the baseline clinical measures across disease groups, for example, hATTR patients had higher bilirubin levels and slightly lower baseline TTR levels; however, there was a larger range of baseline TTR levels in the hATTR population. The hATTR patients, as describe earlier, were in general an older population that had lower creatinine clearance levels compared to healthy volunteers. Given these correlations between disease status and other variables, disease status was selected preferentially as the covariate of interest if two covariates appeared to be correlated in the final model. 


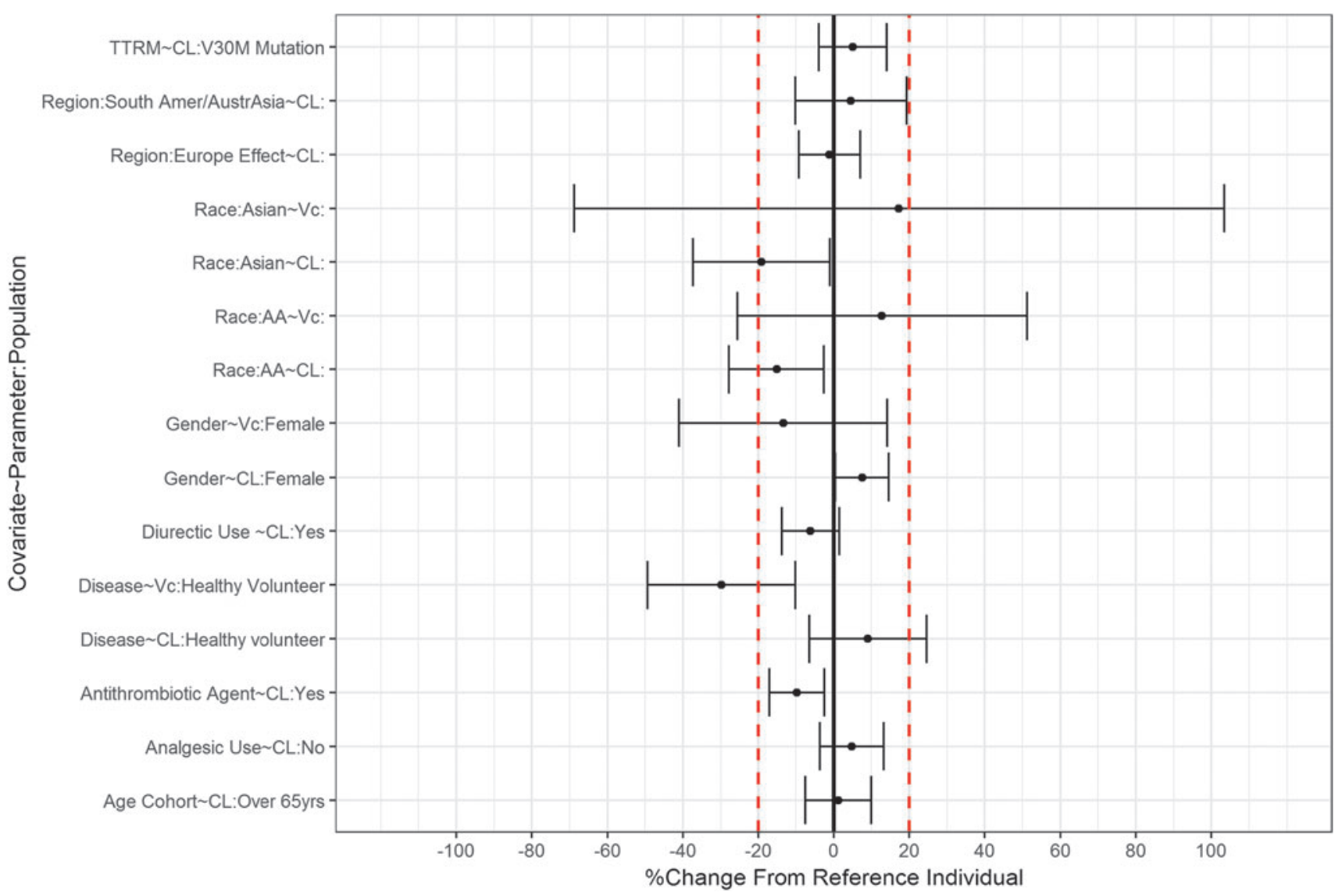

FIG. 2. Clinical significance testing for categorical covariates in full model: distribution of percentage change from reference individual. Vertical red dashed lines, boundaries representing the $20 \%$ clinical significant test; closed circle, median percentage change from the reference category; Whiskers, 5th and 95th CI of the percentage change from the reference category. $\mathrm{Cl}$, apparent clearance, $\mathrm{CL} / \mathrm{F}$; Vc, apparent central volume, Vc/F; Amer for America, AA for African American.

Other statistically and clinically insignificant covariates were excluded from the model through backward elimination process, which included age effects ( $>65$ years old), sex, race, baseline eGFR, baseline albumin, baseline bilirubin normalized by ULN, baseline ALT, baseline TTR, TTR V30M mutation, concomitant use of diuretics, antithrombotic, and non-NSAID analgesics, and region of the world. It was noted that the apparent differences in observations due to race should be interpreted with caution; as $87.6 \%$ of the subjects were Caucasians, these apparent differences could be the result of small sample size.

The final model was qualified using visual predictive checks and nonparametric bootstrap analysis. As indicated by the goodness-of-fit, residual diagnostic plots and prediction corrected visual predictive checks, the model provided an adequate characterization of the observed inotersen plasma concentration-time course (Supplementary Figs. S3 and S4). The structural model parameters were generally estimated with good precision (\% RSE $<30 \%$ ), except for the covariate effect of disease status on CL/F and $V_{\mathrm{c}} / F$. The final model parameter estimates are in good agreement with bootstrapping results and well within the $95 \%$ CIs of parameter estimates for each parameter (Table 2). None of the random effects had shrinkage values $>30 \%$ or had the center of their distributions statistically different from zero.
Based on final estimated population PK parameters, the calculated alpha and beta (terminal) half-lives are $3.91 \mathrm{hs}$ and 26.9 days, respectively, consistent with the PK property of inotersen being biphasic profile with an initial rapid disposition phase followed by a slower elimination phase with apparent terminal elimination half-life of $\sim 4$ weeks. The calculated accumulation ratio based on beta and dosing interval is 6.07, in agreement with the simulation results (Table 4).

\section{Population PD modeling}

As described earlier, there was a slight difference in baseline TTR levels between healthy volunteers and hATTR patients; therefore, disease effect on baseline was added as a covariate to the estimated baseline. V30M mutation showed no effect on the estimated $\mathrm{IC}_{50}$ value for hATTR patients.

The robustness and precision of the final model parameter estimates were confirmed by the $95 \%$ CIs from the bootstrap results (Table 3 ). The overall population $I_{\max }$ and $\mathrm{IC}_{50}$, together with $95 \% \mathrm{CI}$, was estimated to be 0.913 (0.899-0.925) and 9.07 (8.08-10.1) ng/mL, respectively. The final model was qualified using visual predictive checks and nonparametric bootstrap analysis. As indicated by the goodness-of-fit, residual diagnostic plots and prediction corrected visual predictive 


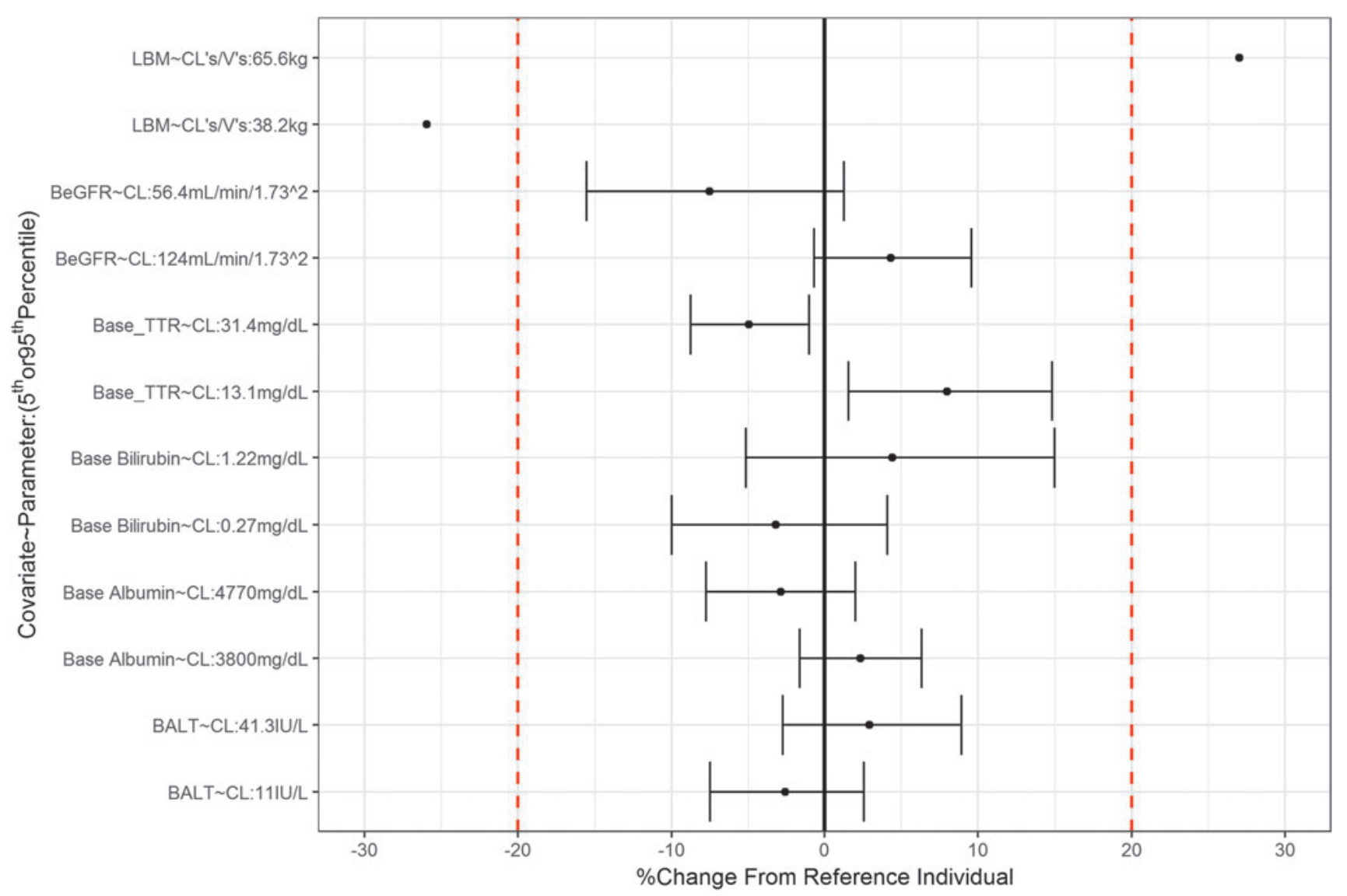

FIG. 3. Clinical significance testing for continuous covariates in full model: distribution of 5th and 95th confidence intervals of percentage change from reference individual. Y Axis Labels, values of the $5^{\text {th }}$ and the $95^{\text {th }}$ percentile of the continuous covariate being tested are displayed as separate categories on the X-axis; Vertical Red Dashed Lines, boundaries representing the $20 \%$ cinicalsignificanfe test; Closed Circle, median percentage change from the reference category Whisker. $5^{\text {th }}$ and $95^{\text {th }}$ percentile of the percentage change from the reference category. $\mathrm{B}$ or $\mathrm{Base}$ refter to baseline. $\mathrm{Cl}$, apparent clearance, CL/F and Apparent inter-compartmental clearence Q/F; Vc, apparent central volume, Vc/F; Vp, periphereal volumes $\mathrm{Vp} / \mathrm{F}$.

Table 2. Parameter Estimates for Final Population Pharmacokinetic Model

\begin{tabular}{|c|c|c|c|c|}
\hline Parameter & Covariate model & Estimate & $\% R S E$ & $95 \% C I$ \\
\hline Clearance, CL (L/h) & & 3.4 & 5.23 & $3.05-3.75$ \\
\hline Central volume, $V_{\mathrm{c}}(\mathrm{L})$ & & 20.7 & 7.87 & $17.5-23.9$ \\
\hline Absorption rate constant, $K_{\mathrm{a}}(1 / \mathrm{h})$ & & 0.261 & 7.73 & $0.222-0.301$ \\
\hline Peripheral volume, $V_{\mathrm{p}}(\mathrm{L})$ & & 230 & 13.8 & $167-292$ \\
\hline Intercompartmental clearance, $Q(\mathrm{~L} / \mathrm{h})$ & & 0.266 & 9.61 & $0.216-0.315$ \\
\hline Dose effect $\sim \mathrm{CL}$ & Exponential & 3.97 & 25.2 & $2.01-5.93$ \\
\hline Lean body mass $\sim \mathrm{CL} / Q$ & Power-centered on median & 1 FIXED & NA & NA \\
\hline Lean body mass $\sim V_{\mathrm{c}} / V_{\mathrm{p}}$ & Power-centered on median & 1 FIXED & NA & NA \\
\hline Disease $\sim \mathrm{CL}$ & Proportional & 0.111 & 46.9 & $0.009-0.214$ \\
\hline Disease $\sim V_{\mathrm{c}}$ & Proportional & -0.284 & 31.4 & -0.459 to -0.109 \\
\hline Covariance, $V_{\mathrm{c}} \sim \mathrm{CL}$ & & 0.049 & 48.4 & $0.003-0.096$ \\
\hline Covariance, $\mathrm{CL} \sim V_{\mathrm{p}}$ & & -0.145 & 27.1 & -0.222 to -0.068 \\
\hline$\omega^{2} V_{\mathrm{c}}$ & & 0.335 & 27 & $0.158-0.512$ \\
\hline$\omega^{2} \mathrm{CL}$ & & 0.071 & 21.3 & $0.041-0.101$ \\
\hline$\omega^{2} V_{\mathrm{p}}$ & & 0.677 & 21 & $0.399-0.955$ \\
\hline Residual variability, $\sigma^{2}$ & Log additive error & 0.168 & 5.29 & $0.151-0.186$ \\
\hline
\end{tabular}

Base model did not have any covariates; reduced and final models had covariates removed as result of analysis. Covariances of parameter displayed as " $\omega^{2}$ parameter."

$\%$ RSE, relative standard error (\%) calculated as standard error divided by parameter estimate; CI, confidence interval; NA, not applicable; AUC, area under the curve. 
Table 3. Final Population Pharmacokinetic/Pharmacodynamic Model Parameter Estimates

\begin{tabular}{lccc}
\hline Parameter & Units & Final model estimates & $95 \%$ CI \\
\hline$I_{\max }$ & & 0.913 & $(0.899-0.925)$ \\
$\mathrm{IC}_{50}$ & $\mathrm{ng} / \mathrm{mL}$ & 9.07 & $(8.08-10.1)$ \\
Estimated baseline & $\mathrm{mg} / \mathrm{dL}$ & 20.4 & $(19.7-21.1)$ \\
$\sim$ Disease effect on baseline (healthy volunteers) & & 0.269 & $(0.178-0.360)$ \\
$k_{\text {out }}$ & $\mathrm{h}^{-1}$ & 0.00308 & $(0.00289-0.00327)$ \\
$\omega^{2}$ IC50 & & 0.953 & $(-0.297$ to -0.0669$)$ \\
Covariance, IC50 $\sim k_{\text {out }}$ & & -0.182 & $(0.187-0.389)$ \\
$\omega^{2} k_{\text {out }}$ & & 0.288 & $(0.0404-0.0670)$ \\
$\omega^{2}$ Baseline & & 0.0537 & $(0.117-0.143)$ \\
Residual variability (log space), (SD) & & 0.13 & $(1.26-1.45)$ \\
Proportional error & & 1.35 & \\
Additive error & & & \\
\hline
\end{tabular}

Disease effect covariate was the proportional shift in baseline TTR levels of healthy volunteers compared to hATTR patients. The 95\% CI was determined from the successful covariance step of the PD model.

PD, pharmacodynamic; hATTR, hereditary transthyretin amyloidosis.

check plots (1,000 simulations), the model provided an adequate characterization of the observed TTR concentrationtime course (Supplementary Figs. S5 and S6). The 5th, 50th, and 95th percentiles of the simulations were consistent with the observed data. In addition, the observed data (blue circles) were well within $90 \%$ of the prediction interval. Although the prediction variability was larger than the observed data, the predicted median values were mainly used when comparing various simulated dosing regimens. Therefore, these support the adequacy of the model and its suitability to investigate alternative dosing regimens using simulation.

\section{Simulations}

The final population PK and PD model was used to simulate four different dosing regimens of inotersen, from which the geometric mean along with $90 \% \mathrm{CI}$ was calculated for $\mathrm{AUC}_{0-}$ ${ }_{\tau}, C_{\max }$, and $C_{\text {trough }}$ for day 1 and steady state (Table 4). Plots of the median plasma trough concentration and TTR profiles over time along with $90 \%$ prediction interval were created for each dosing regimen (Figs. 4 and 5). PK simulations showed that there was little accumulation in $\mathrm{AUC}_{0-\tau}$ and $C_{\max }$ for all dosing regimens. There was also little difference in $\mathrm{AUC}_{0-\tau}$ between the different 300-mg regimens, while the $150-\mathrm{mg}$ regimen produced $\mathrm{AUC}_{0-\tau}$ approximately half of the 300-mg regimens. A similar trend was seen with $C_{\max }$.

The use of $300 \mathrm{mg}$ loading doses achieved trough levels of $\sim 65.1 \%$ and $85.6 \%$ of the steady-state level after 1 and 3 months of $300 \mathrm{mg}$ QW treatment, compared with $47.4 \%$ and $81.5 \%$, respectively, without the use of loading doses. At steady state, the $C_{\text {trough }}$ levels following $300 \mathrm{mg}$ QOW and $150 \mathrm{mg}$ QW treatment regimen were 14.8 (13.3-16.8) ng/mL and $17.2(15.5-19.1) \mathrm{ng} / \mathrm{mL}$, respectively, approximately one half of the $C_{\text {trough }}$ level [34.4 (31.0-38.2) ng/mL] following $300 \mathrm{mg}$ QW treatment regimen. Loading-dose regimen did not alter steady-state trough levels, but reduced time to reach approximately two-thirds of the steady-state $C_{\text {trough }}$ ( $\sim 23 \mathrm{ng} / \mathrm{mL}$ ) level from 2 months to 1 month (Fig. 4).

Similarly, the use of $300 \mathrm{mg}$ loading doses followed by $300 \mathrm{mg}$ QW achieved mean percent change from baseline TTR levels of approximately $-45.1 \%,-57.5 \%$, and $-69.0 \%$ at 2 weeks, 1 month, and 3 months, respectively, compared to approximately $-34.8 \%,-51.2 \%$, and $-67.9 \%$ with no loading doses (Fig. 5; Table 5). A dosing regimen of $150 \mathrm{mg}$ QW resulted in mean percent change from baseline TTR levels of approximately $-28.1 \%,-42.6 \%$, and $-58.6 \%$ at 2 weeks, 1 month, and 3 months, respectively. For $300 \mathrm{mg}$ QOW, the values were $-24.7 \%,-38.0 \%$, and $-53.8 \%$, respectively.

Table 4. Simulated Measures of Exposure for Additional Dosing Regimens

\begin{tabular}{lcccc}
\hline Regimen & Day/type & $A U C_{O-\tau}(\mu g \cdot h / m L)$ & $C_{\text {max }}(\mu g / m L)$ & $C_{\text {trough }}(\mathrm{ng} / \mathrm{mL})$ \\
\hline $300 \mathrm{mg}$ QW & 1 & $84.4(77.6-91.6)$ & $6.35(5.61-7.17)$ & $5.62(4.92-6.24)$ \\
$300 \mathrm{mg}$ QOW & 1 & $85.5(78.4-92.5)$ & $6.33(5.66-7.18)$ & $4.28(3.82-4.74)$ \\
Loading + 300 mg QW & 1 & $85.5(78.5-92.8)$ & $6.36(5.76-7.26)$ & $25.7^{\mathrm{a}}(20.9-31.8)$ \\
$150 \mathrm{mg}$ QW & 1 & $42.3(38.8-45.9)$ & $3.16(2.81-3.57)$ & $2.81(2.45-3.11)$ \\
$300 \mathrm{mg}$ QW & 449 & $89.9(82.4-97.4)$ & $6.39(5.65-7.20)$ & $34.3(31.0-38.2)$ \\
$300 \mathrm{mg}$ QOW & 449 & $90.1(82.6-97.5)$ & $6.34(5.67-7.20)$ & $14.8(13.3-16.8)$ \\
Loading +300 mg QW & 449 & $90.0(82.8-97.8)$ & $6.34(5.72-7.24)$ & $34.3(30.6-38.3)$ \\
$150 \mathrm{mg}$ QW & 449 & $45.0(41.4-48.8)$ & $3.18(2.83-3.59)$ & $17.2(15.5-19.1)$ \\
$300 \mathrm{mg}$ QW & AR & $1.06(1.06-1.07)$ & $1.01(1.00-1.01)$ & $6.11(5.26-7.30)$ \\
$300 \mathrm{mg}$ QOW & AR & $1.05(1.05-1.06)$ & $1.00(1.00-1.00)$ & $3.45(2.99-4.08)$ \\
Loading +300 mg QW & AR & $1.05(1.05-1.06)$ & $0.996(0.991-0.999)$ & $\mathrm{NA}$ \\
$150 \mathrm{mg}$ QW & AR & $1.06(1.06-1.07)$ & $1.01(1.00-1.01)$ & $6.14(5.27-7.26)$ \\
\hline
\end{tabular}

Data presented are geometric mean $(90 \% \mathrm{CI})$.

${ }^{\text {a }} C_{\text {trough }}$ after the dose on day 1 is $48 \mathrm{~h}$ time point during loading dose period.

AUC, area under cureve; QW, once weekly; QOW, once every other week; AR, accumulation ratio. 


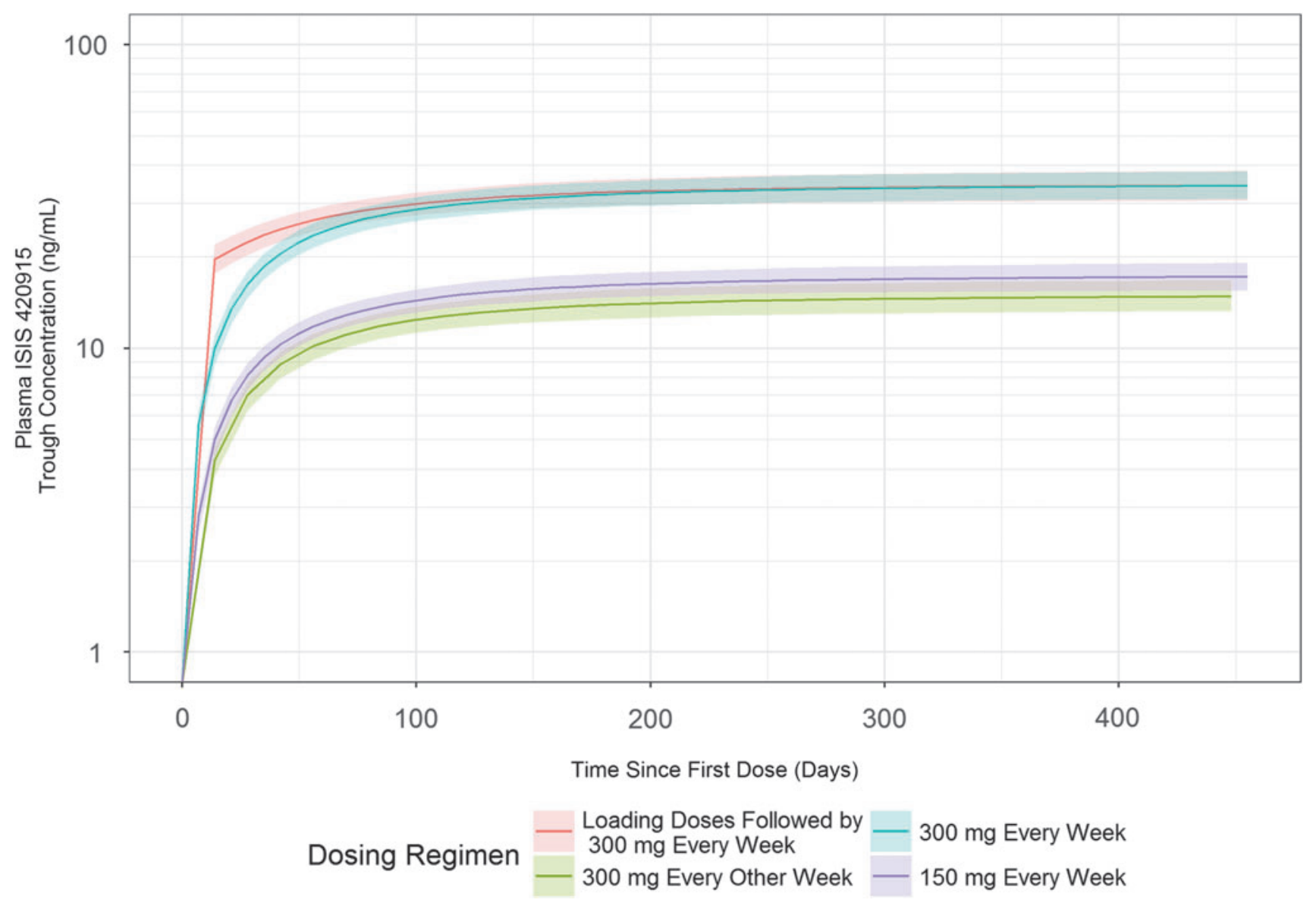

FIG. 4. Simulated plasma trough concentration profile for ISIS 420915 for various dosing regimens.

Overall, $300 \mathrm{mg}$ loading doses followed by $300 \mathrm{mg}$ QW would result in a slightly faster onset of TTR reduction than without loading doses, and the same predicted geometric mean reduction of approximately $-71.9 \%$ at steady state.

\section{Discussion}

We have developed and reported the first population PK and PK/PD model for an ASO therapeutic, inotersen. Inotersen PK is well described by a two-compartment linear PK model, and its pharmacological effect on TTR is well described using an inhibitory effect indirect response PD model, where TTR formation is modulated by inotersen exposure.

Out of the demographic parameters evaluated, including age, gender, body weight, and race, only body weight or body size was shown to be a significant covariate on inotersen clearance and volume of distribution. However, its overall impact on PK exposure $\left(C_{\max }, \mathrm{AUC}\right.$, or $\left.C_{\text {trough }}\right)$ appeared to be small $(<30 \%)$ by body weight and $38 \%$ by LBM. Nonetheless, the clinical relevance of body weight on plasma exposure, selected safety (platelet), and PD (TTR reduction) measures was further evaluated by graphical analysis. Results showed a weak association between platelet reduction and inotersen plasma exposures $\left(C_{\max }\right.$ and AUC), but less with $C_{\text {trough }}$ (Supplementary Fig. S7) among patients at the same dose level. Similarly, a weak association was observed between body weight and platelet reduction, but not with TTR reduction (internal data). These results suggest that dose reduction, if warranted, would be helpful in reducing the risk of platelet reduction without comprising the PD activity.

The clearance and central volume of inotersen were not affected by baseline TTR level or TTR mutation. However, disease status was retained as a covariate on central compartment clearance and central volume. Nonetheless, the contribution of the disease status on PK was small. For example, the population typical value for central clearance $(\mathrm{CL} / F)$ was 3.40 and $3.18 \mathrm{~L} / \mathrm{h}$ in patients with hATTR-PN, based on post hoc analysis, while the apparent volume of distribution $\left(V_{\mathrm{ss}} / F\right)$ was estimated to be $250.7 \mathrm{~L}$ as the typical value and $293 \mathrm{~L}$ in patients with hATTR.

Even though the PK of inotersen is not affected by hepatic function, no conclusions can be drawn for patients with hepatic impairment due to the absence of such population in the clinical program. The population PK analysis showed that renal function, as represented by baseline eGFR, is not a statistically significant covariate for inotersen clearance, in the range of $41.3-127 \mathrm{~mL} / \mathrm{min} / 1.73 \mathrm{~m}^{2}$. Thus, no dose adjustment is necessary in patients with mild-to-moderate renal impairment. Given the lack of experience in patients with low moderate and severe renal impairment for inotersen, no conclusions on the safety in such populations can be drawn, even though the PK of inotersen is expected to be similar across a range renal function impairment based on almost exclusive clearance of inotersen through ubiquitous nucleases in tissues.

Commonly used concomitant medications in the Phase 2/3 study included diuretics (30.5\%), antithrombotic (36.4\%), 


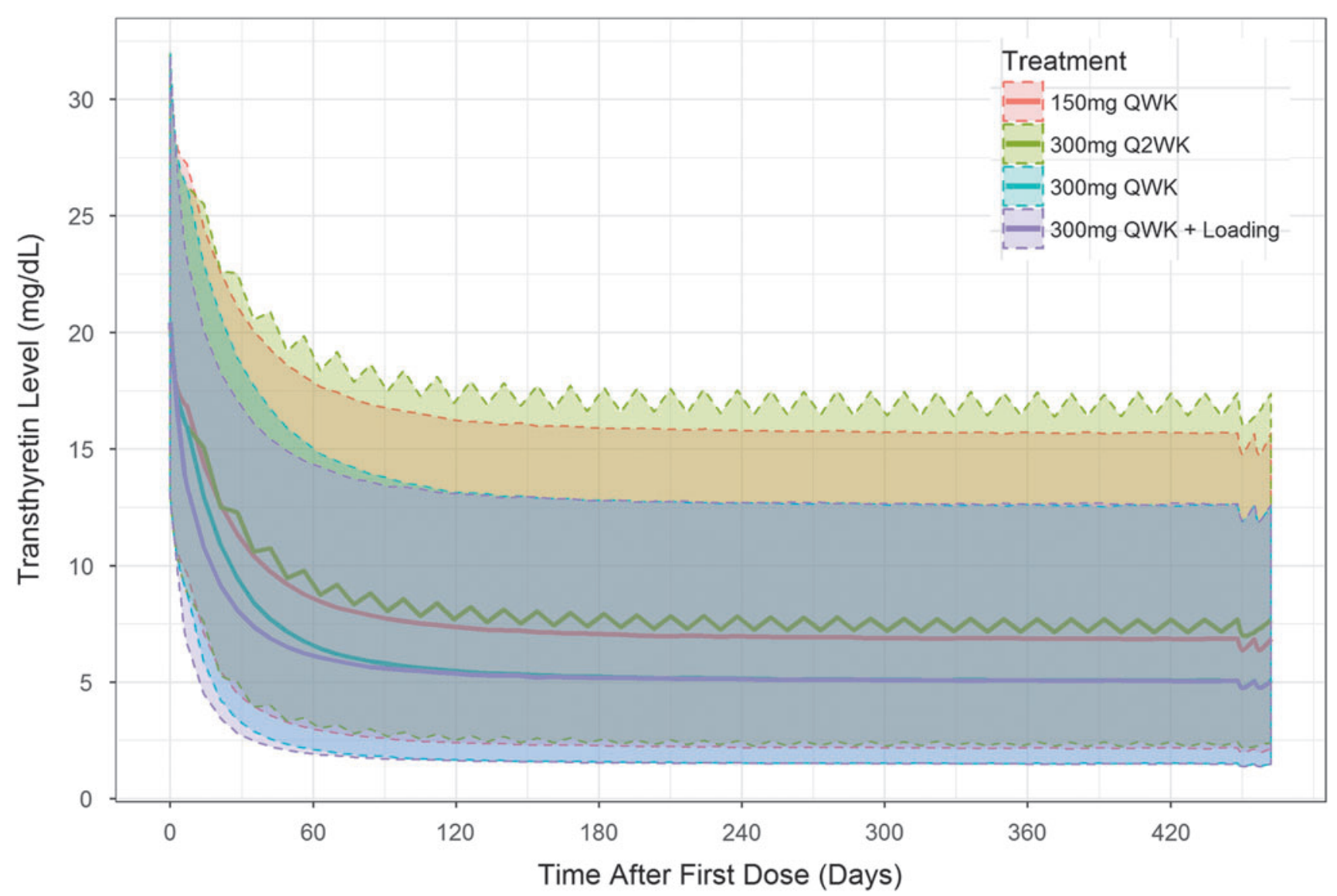

FIG. 5. Predicted transthyretin levels for various simulated dosing regimens. Solid lines represent medians, and shaded regions represent $90 \%$ PI.

Table 5. Simulated Transthyretin Levels Over Time for Various Dosing Regimens

\begin{tabular}{|c|c|c|c|}
\hline Dose & Day & TTR level $(\mathrm{mg} / \mathrm{dL})$ & TTR \% change from baseline (\%) \\
\hline \multirow[t]{6}{*}{$300 \mathrm{mg}$ QW } & Baseline & $20.4(19.7-21.2)$ & NA \\
\hline & Day 7 & $15.7(15.0-16.4)$ & $-20.2(-23.0$ to -17.5$)$ \\
\hline & Day 14 & $12.4(11.8-13.1)$ & $-34.8(-37.9$ to -32.0$)$ \\
\hline & Day 28 & $8.91(8.33-9.53)$ & $-51.2(-54.1$ to -48.4$)$ \\
\hline & Day 85 & $5.56(5.12-6.02)$ & $-67.9(-70.2$ to -65.5$)$ \\
\hline & Day (steady state) & $4.82(4.41-5.22)$ & $-71.9(-74.0$ to -69.7$)$ \\
\hline \multirow[t]{6}{*}{$300 \mathrm{mg}$ QW with loading dose } & Baseline & $20.4(19.7-21.1)$ & NA \\
\hline & Day 7 & $13.0(12.4-13.7)$ & $-32.5(-35.3$ to -29.2$)$ \\
\hline & Day 14 & $10.3(9.72-11.0)$ & $-45.1(-47.8$ to -42.3$)$ \\
\hline & Day 28 & $7.67(7.10-8.26)$ & $-57.5(-60.4$ to -54.8$)$ \\
\hline & Day 85 & $7.39(6.84-7.96)$ & $-69.0(-71.1$ to -66.8$)$ \\
\hline & Day (steady state) & $4.78(4.39-5.22)$ & $-71.9(-74.1$ to -69.8$)$ \\
\hline \multirow[t]{6}{*}{$300 \mathrm{mg}$ Q2W } & Baseline & $20.4(19.7-21.2)$ & NA \\
\hline & Day 7 & $15.7(15.0-16.4)$ & $-20.2(-23.0$ to -17.5$)$ \\
\hline & Day 14 & $14.6(13.9-15.3)$ & $-24.7(-27.8$ to -21.8$)$ \\
\hline & Day 28 & $11.7(11.0-12.6)$ & $-38.0(-41.1$ to -35.0$)$ \\
\hline & Day 85 & $8.29(7.70-8.92)$ & $-53.8(-56.7$ to -50.9$)$ \\
\hline & Day (steady state) & $7.24(6.67-7.82)$ & $-58.8(-61.5$ to -56.1$)$ \\
\hline \multirow[t]{6}{*}{$150 \mathrm{mg}$ QW } & Baseline & $20.4(19.7-21.2)$ & NA \\
\hline & Day 7 & $16.6(15.9-17.4)$ & $-15.9(-18.6$ to -13.3$)$ \\
\hline & Day 14 & $13.9(13.3-14.6)$ & $-28.1(-31.5$ to -25.3$)$ \\
\hline & Day 28 & $10.8(10.1-11.4)$ & $-42.6(-45.6$ to -39.6$)$ \\
\hline & Day 85 & $7.39(6.84-7.96)$ & $-58.6(-61.2$ to -56.0$)$ \\
\hline & Day (steady state) & $6.47(5.95-6.98)$ & $-63.1(-65.7$ to -60.5$)$ \\
\hline
\end{tabular}


and non-NSAID analgesics (83.4\%) in patients with hATTR. None of these concomitant medications showed any clinically significant effect on clearance of inotersen and was not included in the final population PK model. Therefore, dose adjustment for inotersen in consideration of PK interaction when used concomitantly with these agents is not necessary, consistent with different metabolism pathways for inotersen (ubiquitous nuclease mediated metabolism) and small molecule drugs (mainly metabolized by cytochromes P450 [CYPs]).

There was a slight difference between TTR baseline levels for healthy volunteers and patients with hATTR; therefore, disease effect on TTR baseline was added as a covariate to the estimated baseline in the population PK/PD model. There was little difference in the estimated $\mathrm{IC}_{50}$ for patients with $(8.17 \mathrm{ng} / \mathrm{mL})$ and without $(9.19 \mathrm{ng} / \mathrm{mL}) \mathrm{V} 30 \mathrm{M}$ mutation. The model determined $k_{\text {out }}$ was $0.00308 / \mathrm{h}$, corresponding to a half-life of $\sim 9$ days, which is much longer than the $\sim 2$-day half-life of plasma TTR (aka, prealbumin) as reported in the literature (turnover rate of 0.296/day) [9]. This discrepancy may be explained by the PD profiles being driven by inotersen $\mathrm{PK}$, which is the rate-limiting step.

The change from baseline for mNIS +7 score on week 66 (day 449)plotted against day 85 TTR levels showed clear distinction between placebo and inotersen groups, and the majority of patients on inotersen had $>50 \%$ reduction in TTR levels (Supplementary Fig. S8a). Similarly, the change from baseline for Norfolk QoL-DN score on week 66 plotted against day 85 TTR levels showed similar results as noted for mNIS +7 (Supplementary Fig. S8b). These results suggest that a TTR reduction of approximately $>50 \%$ is associated with clinical benefit.

TTR reduction is dose- and exposure dependent. PK/PD simulations showed that $300 \mathrm{mg}$ weekly dosing would produce the best numeric TTR reduction; however, the range of TTR reduction is largely overlapping among the three dosing regimens simulated, that is, $300 \mathrm{mg}$ weekly, $300 \mathrm{mg}$ biweekly, and $150 \mathrm{mg}$ weekly, suggesting that the two lower doses would be effective in TTR reduction for the majority of patients. Loading dose shortened the time to steady state, but showed little effect on TTR reduction at 3 months and beyond. Therefore, loading dose may not be necessary for chronic treatment with inotersen.

In summary, the population PK and PK/PD models developed in this study may be useful for dose optimization in patients receiving inotersen and for development of an inotersen follow-on in the same patient population.

\section{Acknowledgments}

The authors thank Drs. Hoke for his scientific discussion, Angela Colabucci and Robert Saunders for their administrative support.

\section{Author Disclosure Statement}

R.Z.Y., S.H., R.G., B.M., S.H., and Y.W. are employees of Ionis Pharmaceuticals, Inc. and hold stock in the company. E.J.A. is an employee of Avidity Biosciences, San Diego, CA and was a former employee of Ionis Pharmaceuticals and holds stock in the company. J.W.C. is currently an employee of GlaxoSmithKline and was employed with Parexel at the time of work.

\section{Funding Information}

Ionis Pharmaceuticals, Inc. supported this work.

\section{Supplementary Material}

Supplementary Figure S1

Supplementary Figure S2

Supplementary Figure S3

Supplementary Figure S4

Supplementary Figure S5

Supplementary Figure S6

Supplementary Figure S7

Supplementary Figure S8

\section{References}

1. Benson MD, M Waddington-Cruz, JL Berk, M Polydefkis, PJ Dyck, AK Wang, V Plante-Bordeneuve, FA Barroso, G Merlini, et al. (2018). Inotersen treatment for patients with hereditary transthyretin amyloidosis. N Engl J Med 379:22-31.

2. Quintas A, DC Vaz, I Cardoso, MJ Saraiva and RM Brito. (2001). Tetramer dissociation and monomer partial unfolding precedes protofibril formation in amyloidogenic transthyretin variants. J Biol Chem 276:27207-27213.

3. Plante-Bordeneuve V and G Said. (2011). Familial amyloid polyneuropathy. Lancet Neurol 10:1086-1097.

4. Coelho T, BG Ericzon, R Falk, DR Grogan, S Ikeda, M Maurer, V Planté-Bordeneuve, O Suhr and P Trigo. (2008). A Physician's Guide to Transthyretin Amyloidosis. Clarkston, MI: Amyloidosis Foundation. pp 1-16.

5. Liefaard L, Grundy J, Williams N, Shenker A, and Chen C. (2013). Predicting levels of pharmacological response in long-term patient trials based on short-term dosing PK and biomarker data from healthy subjects. Population Approach Group Europe (PAGE) Meeting: Abstract III-15, pp 228. https://www.page-meeting.org/page/page2013/PAGE2013 ProgramAndAbstracts 130604.pdf

6. Ionis-Pharmaceuticals. (2018). Full prescribing information for Tegsedi, https://accessdata.fda.gov/drugsatfda_docs/label /2018/211172lbl.pdf accessed January 17, 2020.

7. Yu RZ, B Baker, A Chappel, RS Geary, E Chueng and AA Levin. (2002). Development of an ultrasensitive noncompetitive hybridization-ligation enzyme-linked immunosorbent assay for the determination of phosphorothioate oligodeoxynucleotide in plasma. Anal Biochem 304:19-25.

8. Gastonguay M. (2011). Full covariate models as an alternative to methods relying on statistical significance for inferences about covariate effects: a review of methodology and 42 case studies. Abstract 2229, p 20. www.page-meeting.org/?abstract=2229.

9. Socolow EL, KA Woeber, RH Purdy, MT Holloway and SH Ingbar. (1965). Preparation of I-131-labeled human serum prealbumin and its metabolism in normal and sick patients. J Clin Invest 44:1600-1609.

Address correspondence to: Rosie Z. Yu, PhD

Ionis Pharmaceuticals, Inc. 2855 Gazelle Court Carlsbad, CA 92010

USA

E-mail: ryu@ionisph.com

Received for publication September 3, 2019; accepted after revision January 13, 2020; published online April 15, 2020. 